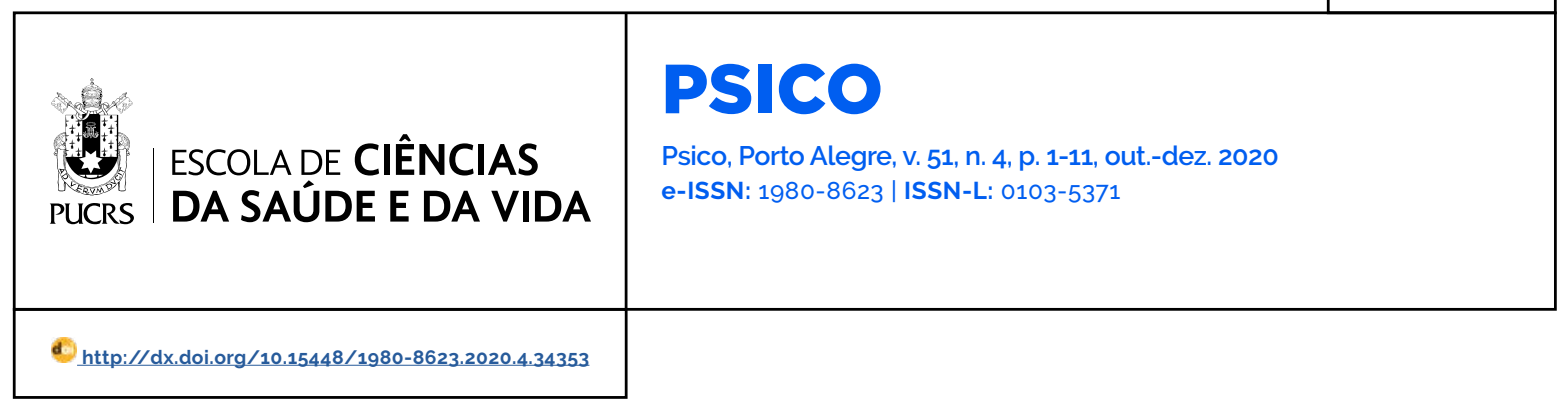

ARTIGO

\title{
Fatores de risco e proteção e sintomas de depressão na adolescência
}

\author{
Risk and protective factors and symptoms of depression in adolescence \\ Factores de riesgo y protección y sintomas de depresión en la adolescência
}

\section{Laís Santos-Vitti ${ }^{1}$ \\ orcid.org/0000-0001-7246-7476 \\ laiss.santos9597@gmail.com}

\section{André Faro ${ }^{2}$}

orcid.org/0000-0002-7348-6297

andre.faro.ufs@gmail.com

\section{Makilim Nunes \\ Baptista ${ }^{3}$}

orcid.org/0000-0001-6519-254X

makilim.baptista@usf.edu.br

Recebido em: 4 jun. 2019

Aprovado em: 14 set. 2020

Publicado em: 15 mar. 2021.

\section{(c) (i)}

Artigo está licenciado sob forma de uma licença Creative Commons Atribuição 4.0 Internacional.
Resumo: Avaliou-se a relação entre fatores de proteção e risco e sintomas depressivos na adolescência. Foi realizado um estudo transversal com amostra de 388 adolescentes de Sergipe, estudantes do ensino médio. Utilizaram-se (a) um questionário sociodemográfico/escolar e (b) as escalas da Bateria de Avaliação de Indicadores de Depressão Infantojuvenil (BAID-IJ). Os dados foram analisados por meio do teste de Regressão Logística Multinomial. Observou-se que o sexo feminino $(O R=6,1)$ e ter idade igual ou superior a 16 anos $(O R=3,3)$ aumentaram consideravelmente as chances de os adolescentes pontuarem no estrato de elevada pontuação quanto à sintomatologia depressiva. Baixa pontuação em autoconceito $(O R=5,7)$, elevado desamparo $(O R=12,3)$, elevada solidão $(O R=7,0)$ e elevada desesperança $(O R=2,8)$ exibiram impacto significativo para a presença de sintomas de depressão. Acredita-se que tais achados podem auxiliar na elaboração de intervenções eficazes frente aos sintomas depressivos na adolescência.

Palavras-chave: fator de risco e proteção, depressão, adolescentes

Abstract: In this research we evaluated the relationship between protection factors and risk factors with depressive symptoms in adolescence. For that, a cross-sectional study was conducted with 388 Sergipe high school students. A socio-demographic / school questionnaire was used and the scales of the Assessment of Childhood Depression Indicators (BAID-IJ). Data were analyzed using the Multinomial Logistic Regression test. We observed that the female gender $(O R=6.1)$ and age 16 years or older $(O R=3.3)$ significantly increased the odds of the adolescents score in the high stratum of depressive symptomatology. Low self-concept scores (OR $=5.7$ ), high helplessness ( $O R=12.3$ ), high loneliness (OR $=7.0)$ and high hopelessness $(O R=2.8)$ had a significant impact on the presence of symptoms of depression. It is believed that such findings may aid in the development of effective interventions for depressive symptoms in adolescence.

Keywords: risk and protection factor, depression, teenagers

Resumen: Se evaluó la relación factores de protección y riesgo y síntomas depresivos en la adolescencia. Fue un estudio transversal con 388 adolescentes en Sergipe (Brasil). Se utilizó un cuestionario sociodemográfico/escolar y las escalas de la Batería de Evaluación de Indicadores de Depresión Infantojuvenil (BAID-IJ). Los datos fueron analizados por intermedio de Regresión Logística Multinomial. El sexo femenino $(O R=6,1)$ y tener edad igual o superior a 16 años $(O R=3,3)$ aumentaron considerablemente las posibilidades de puntuar en el estrato de alta puntuación de la sintomatología depresiva. La baja puntuación en autoconcepto $(\mathrm{OR}=5,7)$, elevado desamparo $(\mathrm{OR}=12,3)$, elevada soledad $(\mathrm{OR}=7,0)$, y elevada desesperanza $(\mathrm{OR}=2,8)$ mostraron impacto significativo en la presencia de sintomas de la depresión. Se cree que estos hallazgos pueden ayudar en la elaboración de intervenciones eficaces frente a los sintomas depresivos en la adolescencia. Palabras clave: factore de riesgo y protección, depresión, adolescentes

\footnotetext{
Pontifícia Universidade Católica de Campinas (PUC-Campinas), Campinas, SP, Brasil.

Universidade Federal de Sergipe (UFS), São Cristóvão, SE, Brasil.

Universidade São Francisco (USF), Bragança Paulista, SP, Brasil.
} 
Mais de 300 milhões de pessoas são acometidas pela depressão. Sabe-se que o Brasilé o primeiro país da América Latina e o segundo das Américas, com mais prevalência de depressão (mais de 11 milhões de pessoas (World Health Organization [WHO], 2017). Entre os adolescentes, 13,4\% apresentam alguma doença de ordem psicológica, sendo a depressão e os transtornos de ansiedade os mais comuns (Reardon et al., 2017). A adolescência é um periodo relativamente negligenciado em relação à produção de pesquisas no que concerne à saúde física e mental (Luz et al., 2018). A priori, até a década de 1970, se acreditava que a depressão seria uma doença que não acometeria os adolescentes por esses não terem capacidade de desenvolvê-la (Maughan, Collishaw, \& Stringaris, 2013).

Para a WHO (2017), a depressão é a terceira principal causa de adoecimento entre os adolescentes. A adolescência então parece ser um período decisivo no que tange aos processos de saúde e doença, uma vez que muitos problemas se iniciam nessa fase e se estendem para a idade adulta, tornando-se crônicos (Kaushik, Kostaki, \& Kyriapoulos, 2016; Sagar \& Krishnan, 2017). Além do gasto em saúde, várias consequências negativas podem ser geradas para o adolescente, como a queda no rendimento escolar, menores niveis de satisfação com a vida, mais ansiedade e depressão, entre outros (Das et al., 2016; Kaushik, et al., 2016). Atrelado ao desenvolvimento de crianças e de adolescentes, identifica-se a presença dos chamados fatores de risco e de proteção, que podem dificultar ou potencializar o desenvolvimento das competências necessárias para uma adaptação positiva, e, consequente saúde mental (Luz, Murta, \& Aquino, 2015). Os chamados fatores de risco são características cuja origem pode ser genética, biológica, psicossocial ou até mesmo cultural, que aumentam a vulnerabilidade de alguns sujeitos ao desenvolvimento de problemas comportamentais e emocionais em comparação à população. Fatores de proteção são aspectos que diminuem a vulnerabilidade das pessoas frente aos riscos e a resultados não adaptativos. Com efeito, identificar fatores de proteção e de risco entre os jovens é fundamental para a pre- venção e a alteração do surgimento de doenças e o agravamento delas (Luz et al., 2015).

Sobre os fatores de risco e de proteção, estima-se que metade de todos os transtornos mentais na idade adulta se inicia por volta dos 14 anos de idade, ainda que parte não seja diagnosticada e nem tratada (WHO, 2014). Evidências revelam que a genética e o ambiente social desestruturado atuam como fatores de risco para o desenvolvimento da depressão. Referente às diferenças por sexo, observa-se que as meninas tendem a ter mais sintomas depressivos do que os meninos, fato que pode ser justificado, por exemplo, pelas mudanças físicas, puberdade, amadurecimento precoce e preocupações relacionadas à imagem e a rejeição do grupo (McGuire, McCormick, Koch, \& Mendle, 2019).

Estudos demonstraram que caracteristicas pessoais positivas, tais como a autoestima, autoeficácia e o autoconceito, possibilitam o enfrentamento de adversidades de modo mais adaptativo. Tais características são recursos pessoais que podem ser desenvolvidos e se associam à capacidade individual de controlar e influenciar e ser influenciado pelo ambiente. Em niveis mais elevados, essas caracteristicas se associam a menos estresse e maiores índices de bem-estar subjetivo, atuando assim como fatores protetivos (Freitas, 2016). Nesse contexto, por exemplo a autoeficácia, a autoestima e os afetos positivos podem atuar na remissão de sintomas depressivos entre adolescentes (Yang, Lau, \& Lau, 2018). Outra característica positiva de valor significativo é o autoconceito, de modo que se acredita que baixos niveis de autoconceito podem se associar a altos niveis de depressão (Robles-Piña, 2011).

Em especial, a autoestima costuma estabelecer uma associação negativa com sintomas depressivos, ou seja, reduzida autoestima é considerada um potencial fator explicativo para o maior risco em desenvolver depressão (Orth \& Robins, 2014). A exemplo disso, um estudo com adolescentes brasileiros demonstrou associação negativa entre os niveis de autoestima e sintomas de transtorno mental em geral (Paixão, Patias, \& Dell'Aglio, 2018).

Por sua vez, os aspectos negativos como a desesperança, a solidão e o desamparo podem 
atuar como fatores de risco para o desenvolvimento de sintomas depressivos em adolescentes. Matthews et al. (2016), por meio de um estudo realizado com gêmeos ao longo de 18 anos, mostrou que o isolamento social e a solidão se correlacionaram positivamente e com força moderada. Já a depressão se correlacionou negativa e moderadamente com a solidão, sendo um fator de maior impacto do que o isolamento social.

Para Verma e Gera (2014), o desamparo aprendido seria uma condição negativa para os adolescentes, visto que esse influenciaria a redução de autoconfiança, motivação e até o desempenho deles, podendo expô-los a problemas mais graves, a exemplo da depressão. Waszczuk, Coulson, Gregory e Eley (2016), ao realizarem um estudo sobre a desesperança, com 2619 gêmeos adolescentes em três etapas - a primeira com participantes com média etária de 15 anos, a segunda 17 e a terceira com 20 - concluíram que ela mediou parcialmente a relação entre o estilo atribucional e os sintomas de depressão. Outro achado foi que se observou que o estilo atribucional e a desesperança se correlacionaram moderadamente com sintomas depressivos e ansiosos, tendo a desesperança uma correlação positiva e o estilo atribucional negativa. Assim, estimou-se que quanto maior o nível de desesperança, mais sintomas de ansiedade e de depressão foram encontrados entre os entrevistados.

Por fim, são relevantes as ações de prevenção primária na adolescência no que concerne ao surgimento de doenças, uma vez que isso leva a um melhor prognóstico nos casos preexistentes e à redução das chances de adoecimento em longo prazo (Das et al., 2016; Cho \& Shin, 2013; Sagar \& Krishnan, 2017). Identificar os principais fatores protetivos e de risco associados à predição de sintomas depressivos pode auxiliar na construção de intervenções eficazes e direcionadas, resultando em maior capacidade de adaptação dos adolescentes frente às adversidades. Logo, esta pesquisa objetivou avaliar a capacidade explicativa da autoestima, da autoeficácia, do autoconceito, do desamparo, da solidão e da desesperança em relação à depressão entre adolescentes. Analisou-se, também, a capacidade explicativa de variáveis sociodemográficas e escolares (sexo, idade, religiosidade, desempenho escolar, interação com colegas de classe e relação com professores), juntamente com características pessoais positivas (autoestima, autoeficácia e autoconceito) e negativas (desamparo, solidão e desesperança) em relação aos sintomas depressivos na adolescência.

\section{Método}

\section{Participantes}

A amostra foi do tipo de não probabilístico, por conveniência, composta por 388 adolescentes (59,5\%; $n=231$ meninas), com média de 16,3 anos $(D P=1,17)$, mínimo de 14 e máximo de 19 anos. Todos eram estudantes do Ensino Médio de três escolas públicas, sendo uma da cidade de Aracaju (63.9\%; $n=248$ ) e as outras duas do municipio de Itabaiana (SE) $(36,1 \% ; n=140)$. Quanto à distribuição por série, $49,7 \%(n=193)$ eram alunos do $1 .^{\circ}$ ano, $29,9 \%(n=116)$ do $2 .^{\circ}$ ano e $20,4 \%(n=$ 79) do $3 .^{\circ}$ ano do ensino médio.

\section{Instrumentos}

Foi utilizada a Bateria de Avaliação de Indicadores de Depressão Infantojuvenil (BAID-IJ) de Borges, Baptista e Serpa (2015). A BAID-IJ contém sete escalas, a saber: depressão (18 itens); solidão (13); desamparo (17); autoestima (18); autoconceito (16); desesperança (14); e autoeficácia (15). As escalas têm opções de resposta do tipo Likert com três pontos, variando de: o - "não, nunca"; 1 - "às vezes"; a 2 - "sim, sempre". Os itens avaliam comportamentos, pensamentos e sentimentos de crianças e adolescentes referentes aos últimos 15 dias. As escalas depressão, solidão e desamparo apresentam itens que versam sobre comportamentos, pensamentos e sentimentos escritos com semântica negativa. Em contrapartida, os itens das escalas de autoestima, autoconceito e autoeficácia são compostos por itens cujo enunciado têm semântica positiva. Apenas a escala de desesperança foi construída com itens de conotação negativa (10 itens) e positiva (4 itens), devendo esses últimos ser invertidos para a computação 
do escore do total da escala de desesperança. $O$ escore total da BAID-IJ é gerado mediante a soma de todos os escores obtidos em cada uma das sete escalas. As pontuações mínima e máxima nas escalas da BAID-IJ variam de: depressão (o a 36 pontos); solidão (o a 26 pontos); desamparo (o a 34 pontos); autoestima (o a 36 pontos); autoconceito (o a 32 pontos); desesperança (o a 28 pontos); e autoeficácia (o a 30 pontos). A consistência interna das escalas, aferida por meio do Alfa de Cronbach ( $\alpha$ ), variou de 0,91 a 0,95 em estudo realizado por Cardoso (2018). Na presente investigação foram obtidos valores de Alfa de Cronbach satisfatórios (> 0,60 ) para todas as subescalas (depressão, $\alpha=0,87$; solidão, $\alpha=0,84$; desamparo, $\alpha=0,90$; autoestima, $\alpha=0,91$; autoconceito, $\alpha=0,88$; desesperança, $\alpha=$ 0,82; autoeficácia, $\alpha=0,86$ ).

Utilizou-se também um questionário sociodemográfico/escolar para caracterização do perfil amostral das seguintes variáveis: sexo (masculino ou feminino); idade (inicialmente medida em anos, e, posteriormente estratificada em três grupos etários - 14 e 15, 16 e 17, 18 e 19 anos); avaliação do desempenho escolar lde o (péssimo) a 10 (excelente)]; interação com colegas de classe [de o (péssima) a 10 (excelente)]; religiosidade [de o (nada religioso) a 10 (muito religioso)]; e relação com os professores (muito ruim, ruim, neutra, boa ou muito boa).

\section{Procedimentos}

Inicialmente, obteve-se a autorização da direção das escolas, dos professores em sala e o consentimento dos alunos, que responderam coletivamente e em sala de aula os instrumentos de pesquisa. Foram apresentados aos participantes a BAID-IJ e o questionário sociodemográfico, respectivamente. Em média, o tempo de resposta gasto variou de 20 a 25 minutos. A presente pesquisa foi aprovada pelo Comitê de Ética em Pesquisa com Seres Humanos da Universidade Federal de Sergipe (UFS) (CAAE: 54236016.0.0000.5546)

\section{Análises dos dados}

Realizaram-se análises por meio do Statistical Package for the Social Sciences (SPSS, versão 22). Geraram-se análises exploratórias e descritivas a fim de verificar a distribuição das variáveis em questão, bem como foram executados ajustes no banco para a correção de missings e outliers, ambos com valores inferiores a $5 \%$ na amostra total. Calcularam-se os escores das escalas da BAID-IJ e, posteriormente, obtiveram-se estatísticas descritivas (frequência absoluta e relativa, média e desvio-padrão).

Utilizou-se a Regressão Logística Multinomial (método stepwise) tendo-se como variável dependente a pontuação na escala de depressão da BAID-IJ, que foi estratificada inicialmente em quartis. Depois, os dois estratos de mais baixa pontuação foram reagrupados, gerando um novo estrato (baixa pontuação). Os outros dois estratos restantes foram reclassificados, resultando então em três estratos, a saber: baixa pontuação (de o a 12 pontos); faixa intermediária (13 a 17 pontos); e elevada pontuação (18 pontos ou mais). Foram incluidos no modelo como variáveis explicativas/independentes as demais escalas da BAID-IJ, os dados sociodemográficos (sexo, grupos etários e religiosidade) e os dados escolares (desempenho escolar, interação com colegas e relação com professores).

A variável religiosidade, desempenho escolare interação com os colegas foram agrupadas em dois estratos ("zero a cinco pontos" e "seis pontos ou mais"). Já a variável relação com professores foi reagrupada em "muito ruim, ruim ou neutra" e "relação boa ou muito boa". Após análise dos parâmetros de normalidade da amostra, detectou-se que os fatores de proteção (autoestima e autoconceito) e de risco (desamparo, solidão e desesperança) tiveram uma distribuição não normal e, por isso, foram agrupados em dois grupos a partir da mediana. Somente a autoeficácia foi estratificada em relação à média, pois exibiu distribuição normal.

Como critérios de avaliação de ajuste do modelo foram considerados o Odds Ratio (OR), índice de Nagelkerke (variância explicada) e a capacidade preditiva do modelo (casos corretamente preditos, com valores esperados a partir de 70\%). Além desses, foram avaliados o Goodness of Fit (esperado um valor de Qui-quadrado não significativo), -2 log Likelihood e o AIC (Critério de Informação de Akaike; quanto maior a diferença entre os modelos inicial e final, melhor o modelo final). Vale salientar que o modelo final utilizou como cate- 
goria de referência o grupo de baixa pontuação na escala de depressão da BAID-IJ, dessa forma os grupos de intermediária e elevada pontuação foram comparados ao de baixa pontuação. Ou seja, com a análise foi verificada a probabilidade de pertencer aos estratos de mais alta vulnerabilidade (intermediária e elevada pontuação) em comparação ao de menor vulnerabilidade (baixa pontuação). O nivel de significância adotado para a inclusão no modelo logístico final foi de $p<0,05$.

\section{Resultados}

Em relação ao perfil geral, 59,5\% $(n=231) \mathrm{da}$ amostra foi composta por meninas. A média de idade foi de 16,3 anos ( $D P=1,17$ ), sendo que $25 \%$ $(n=97)$ enquadraram-se no intervalo de 14 e 15 anos, 58,5\% ( $n=227)$ entre 16 e 17 anos, e 16,5\% ( $n=64)$ entre 18 e 19 anos. Sobre a escolaridade dos entrevistados, $49.7 \%(n=193)$ eram do $1 .^{\circ}$ ano, $29,9 \%(n=116)$ do $2 .^{\circ}$ ano e $20,4 \%(n=79)$ do $3^{\circ}$ ano do ensino médio. Em relação às demais variáveis analisadas, $54,5 \%(n=211)$ pontuaram de o a 5 pontos quanto à religiosidade, $65.5 \%(n=$ 254) relataram ter uma relação boa ou muito boa com os professores, $66 \%(n=256)$ qualificaram o nivel de interação com os colegas em classe de 6 a 10 pontos e $64,7 \%(n=251)$ avaliaram o desempenho escolar no estrato de 6 a 10 pontos.

Quanto aos estratos gerados com o escore total da escala de depressão da BAID-IJ, 27,8\% ( $n=108)$ da amostra pontuou maior ou igual a 18 pontos, situando-se no estrato de elevada pontuação. Quase metade da amostra (49,2\%; $n=191)$ pontuou no grupo de baixa pontuação e 22,9\% (n = 89) na faixa intermediária. Quanto aos demais construtos, os valores percentuais da pontuação de cada variável foram: desamparo, 45,6\% $(n=177$; $\geq 10,1$ pontos, Mínimo = 0 e Máximo = 28); solidão, 48,7\% ( $n=189$; $\geq 6$ pontos, Min = o e Max = 18); desesperança, 49,0\% ( $n=190$; $\geq 8$ pontos, $M i n=0$ e Max = 20); autoestima, 46,4\% ( $n=180$; $\geq 26$ pontos, Min = 3 e Max = 36) e autoconceito, 45,1\% ( $n=175$; $\geq 23$ pontos, $\mathrm{Min}=4 \mathrm{e}$ Max $=32$ ). No que se refere à autoeficácia, 52,1\% pontuaram acima da média ( $n$ = 202; $\geq 20,4$ pontos, Min = 7 e Max = 30) (Tabela 1 ).
Tabela 1 - Distribuição dos estratos das Escalas da Bateria de Avaliação de Indicadores de Depressão Infantojuvenil (BAID-IJ)

\begin{tabular}{|c|c|c|}
\hline $\begin{array}{l}\text { Escalas da } \\
\text { BAID-IJ }\end{array}$ & Estratos (pontos) & $F \%(n)$ \\
\hline \multirow{3}{*}{ Depressão } & Baixa pontuação (o a 12) & $49,2(191)$ \\
\hline & Faixa intermediária (13 a 17) & $22,9(89)$ \\
\hline & Elevada pontuação (18 ou mais) & $27.9(108)$ \\
\hline \multirow[b]{2}{*}{ Desamparo } & Até a mediana (o a 11) & \multirow{2}{*}{$\begin{array}{l}54,4 \text { (211) } \\
45,6 \text { (177) }\end{array}$} \\
\hline & $\begin{array}{l}\text { Acima da mediana }(10,10 \mathrm{ou} \\
\text { mais) }\end{array}$ & \\
\hline \multirow{2}{*}{ Solidão } & Até a mediana ( 0 a 5) & $51,3(199)$ \\
\hline & Acima da mediana (6 ou mais) & $48,7(189)$ \\
\hline \multirow{2}{*}{ Desesperança } & Até a mediana (o a 7) & $51,0(198)$ \\
\hline & Acima da mediana (8 ou mais) & $49,0(190)$ \\
\hline \multirow{2}{*}{ Autoestima } & Até a mediana (o a 25) & $53,6(208)$ \\
\hline & Acima da mediana (26 ou mais) & $46,4(180)$ \\
\hline \multirow{2}{*}{ Autoeficácia } & Até a média (o a 20,3) & $52,1(202)$ \\
\hline & Acima da média ( 20,4 ou mais) & $47.9(186)$ \\
\hline \multirow{2}{*}{ Autoconceito } & Até a mediana (o a 22) & 54.9 (213) \\
\hline & Acima da mediana (23 ou mais) & $45,1(175)$ \\
\hline
\end{tabular}

O modelo final de Regressão Logística Multinomial (Tabela 2) obteve solução aceitável $(p<$ 0,001), com aproximadamente $60,0 \%$ de variância explicada (Nagelkerke $=0,584)$ e apresentou capacidade preditiva total de $70 \%$. O valor de Goodness of Fit não foi estatisticamente significativo ( $p=0,307$ ) e os valores de -2 log Likelihood inicial e final foram de 769,003 e 490,820 ( $\triangle$-2ll $=278,183)$ e os valores de $A / C$ inicial e final de 773,003 e $518,820(\triangle A / C=254,183)$.

Quanto ao grupo de pontuação intermediária em comparação ao de baixa pontuação: o sexo feminino $(O R=3,0)$ exibiu três vezes mais chances de pontuar na faixa intermediária do que na faixa de baixa pontuação na escala de depressão da BAID-IJ. Outras variáveis estatisticamente significativas na faixa intermediária foram: ter idade $\geq 16$ anos $(O R=2,8)$; autoconceito até a mediana $(O R=2,8)$; estar acima da mediana na escala de desamparo $(O R=2,4)$; e solidão $(O R=2,7)$. No estrato elevada pontuação foram significativas as variáveis: sexo feminino $(O R=6,1)$; idade $\geq 16$ anos $(O R=3,3)$; pontuar até a mediana na escala de autoconceito $(O R=5,7)$; e estar acima da mediana 
quanto ao desamparo $(O R=12,3)$, à solidão $(O R=$ $7,0)$ e à desesperança $(O R=2,8)$. Vale ressaltar que a variável sexo, ter idade $\geq 16$ anos, desamparo, solidão e autoconceito foram significativos para ambos os grupos da escala de depressão da BAID-IJ (faixa intermediária e elevada pontuação). Desesperança foi um fator significativo apenas para o grupo de elevada pontuação.

Tabela 2 - Regressão Logística Multinomial - Grupo de Referência "Baixa Pontuação [0 a 12 pontos]" - na Escala de Depressão da BAID-IJ

\begin{tabular}{|c|c|c|c|c|}
\hline $\begin{array}{c}\text { Pontuação na Escala de } \\
\text { Depressão }\end{array}$ & Variáveis & Grupo de análise (F\%) & OR $(95 \%$ IC) & $p$ \\
\hline \multirow{5}{*}{$\begin{array}{l}\text { Faixa intermediária } \\
\text { (Pontuação de } 13 \text { a 17) }\end{array}$} & Sexo & $\begin{array}{l}\text { Feminino }(70,8 \%) \\
\text { Masculino }(29,2 \%)\end{array}$ & $\begin{array}{l}3,0(1,7-5,5) \\
1\end{array}$ & $<0,001$ \\
\hline & Idade $\geq 16$ anos & $\begin{array}{l}\text { Sim }(80,9 \%) \\
\text { Não }(19,1 \%)\end{array}$ & $\begin{array}{l}2,8(1,3-5,6) \\
1\end{array}$ & 0,010 \\
\hline & Autoconceito & $\begin{array}{l}\text { Até a mediana }(65,2 \%) \\
\text { Acima da mediana }(34,8 \%)\end{array}$ & $\begin{array}{l}2,8(1,5-4,8) \\
1\end{array}$ & 0,001 \\
\hline & Desamparo & $\begin{array}{l}\text { Acima da mediana }(47,2 \%) \\
\text { Até a mediana }(52,8 \%)\end{array}$ & $\begin{array}{l}2,4(1,2-4,5) \\
1\end{array}$ & 0,009 \\
\hline & Solidão & $\begin{array}{l}\text { Acima da mediana }(57,3 \%) \\
\text { Até a mediana }(42,7 \%)\end{array}$ & $\begin{array}{l}2,7(1,5-4,9) \\
1\end{array}$ & 0,001 \\
\hline \multirow{6}{*}{$\begin{array}{l}\text { Faixa Elevada } \\
\text { (Pontuação de } 18 \text { ou mais) }\end{array}$} & Sexo & $\begin{array}{l}\text { Feminino }(81,5 \%) \\
\text { Masculino }(18,5 \%)\end{array}$ & $\begin{array}{l}6,1(2,7-13,5) \\
1\end{array}$ & $<0,001$ \\
\hline & Idade $\geq 16$ anos & $\begin{array}{l}\text { Sim }(73,1 \%) \\
\text { Não }(26,9 \%)\end{array}$ & $\begin{array}{l}3.3(1,4-7,8) \\
1\end{array}$ & 0,006 \\
\hline & Autoconceito & $\begin{array}{l}\text { Até a mediana }(87,0 \%) \\
\text { Acima da mediana }(13,0 \%)\end{array}$ & $\begin{array}{l}5,7(2,5-12,8) \\
1\end{array}$ & $<0,001$ \\
\hline & Desamparo & $\begin{array}{l}\text { Acima da mediana }(88,9 \%) \\
\text { Até a mediana }(11,1 \%)\end{array}$ & $\begin{array}{l}12,3(5,2-29,4) \\
1\end{array}$ & $<0,001$ \\
\hline & Solidão & $\begin{array}{l}\text { Acima da mediana }(84,3 \%) \\
\text { Até a mediana }(15,7 \%)\end{array}$ & $\begin{array}{l}7,0(3,2-15,6) \\
1\end{array}$ & $<0,001$ \\
\hline & Desesperança & $\begin{array}{l}\text { Acima da mediana }(88,9 \%) \\
\text { Até a mediana }(11,1 \%)\end{array}$ & $\begin{array}{l}2,8(1,2-6,2) \\
1\end{array}$ & 0,014 \\
\hline
\end{tabular}

Nota: Todas as variáveis de cada grupo foram comparadas ao grupo de referência de baixa pontuação na Escala de Depressão da BAID-IJ.

\section{Discussão}

O presente estudo avaliou a associação entre características pessoais positivas, negativas, variáveis sociodemográficas e sintomatologia depressiva em adolescentes. Constatou-se que a mediana da pontuação obtida na escala de depressão da BAID-IJ foi de 13 ( $D P=6,30$ ), ficando um pouco abaixo da pontuação média do escore possivel (18 pontos), uma vez que esse variava de 0 a 36 pontos.

O sexo feminino exibiu três e 6,1 vezes mais chances de pertencer ao estrato de faixa intermediária e elevada pontuação na escala de depressão da BAID-IJ, respectivamente. As mulheres, quando comparadas aos homens, mostraram maior vulnerabilidade frente à depressão, o que corrobora achados relativos ao tema. Estima-se que a razão da depressão entre os sexos seja de aproximadamente 2:1 em termos globais, respectivamente feminino e masculino (Hodes, Walker, Labonté, Nestler, \& Russo, 2016). Sabe-se que no sexo feminino os sintomas depressivos surgem mais cedo, embora a cronicidade se apresente de modo semelhante para ambos os sexos (Salk, Petersen, Abramson, \& Hyde, 2016). Além de a duração dos 
episódios depressivos ser maior entre as meninas, há diferença significativa também em relação à gravidade do curso da depressão entre os adolescentes que iniciaram o transtorno mais cedo (Essau, Lewinsohn, Seeley, \& Sasagawa, 2010). Ter idade igual ou maior que 16 anos aumentou cerca de três vezes a chance de pertencer ao estrato intermediário e alto em termos de pontuação na escala de depressão da BAID-IJ.

Possiveis justificativas associadas às diferenças de gênero e idade podem estar atreladas às mudanças biopsicossociais inerentes à adolescência, como as questões relacionadas à puberdade (McGuire et al., 2019). Uma meta-análise avaliou a razão de chance da ocorrência do transtorno depressivo e constatou que a idade foi o preditor mais forte. Ser adolescente exibiu duas vezes (OR $=2,0$ ) mais chances de desenvolver sintomas depressivos, bem como ter idade entre 13 e 15 anos exibiu aproximadamente quase três vezes mais chances $(O R=2,92)$. Diferenças de gênero em relação à depressão também foram constadas a partir dos 12 anos $(O R=2,37)$, sendo mais acentuada dos 13 aos 15 anos $(O R=3,02)(O R=2,12)$ e para os 16 anos (Salk, Hyde, \& Abramson, 2017). Em resumo, ratificou-se, tanto neste estudo quanto na pesquisa citada, que as mulheres exibiram taxas mais altas de sintomas depressivos em comparação aos homens. Além disso, evidenciou-se também que em relação à idade, as diferenças de gênero quanto à sintomatologia depressiva acentuaram-se entre os adolescentes com 16 anos.

Diferenças significativas em relação ao gênero dos adolescentes também foram observadas em outras pesquisas realizadas no contexto nacional. A exemplo disso, constatou-se em uma amostra de adolescentes do Rio Grande do Sul, maior prevalência de sintomas moderados e graves de ansiedade entre as meninas do que entre os meninos (Grolli, Wagner, \& Dalbosco, 2017). Foram também encontradas diferenças significativas quanto aos sintomas depressivos e ansiosos entre adolescentes do sexo feminino em comparação aos meninos, bem como foi constatada a estabilidade dos sintomas de depressão no decorrer dos três anos escolares correspondentes ao ensino médio (Germain \& Marcotte, 2016).

Quanto ao autoconceito, aqueles que pontuaram até a mediana na escala de autoconceito tinham cerca de três vezes mais chances de pontuar no estrato de faixa intermediária na escala de depressão da BAID-IJ. Já quanto ao grupo de elevada pontuação, as chances aumentaram para quase seis vezes. Esses resultados se assemelham a outros que apontaram que o autoconceito manteve relação negativa com sintomas depressivos, sendo que quanto mais elevado o nível de autoconceito, menos sintomas de depressão (Robles-Piña, 2011). O autoconceito remete à percepção que as pessoas têm de si próprias, as quais são formuladas por meio das experiências com o meio externo e influenciadas por atribuições interna e externas (Shavelson \& Bolus, 1982). Por esse motivo, entende-se que é possivel que se relacione de modo consistente com a depressão em virtude de que, de acordo com a teoria cognitiva da depressão de Beck, crenças negativas e disfuncionais podem ser tanto sintoma quanto causas para o desenvolvimento dos sintomas depressivos, visto que esse transtorno envolve a distorção do mundo e das emoções (Beck, 2008). Nesse sentido, uma possivel justificativa para a relação de baixos niveis de autoconceito e a presença de sintomas depressivos na adolescência pode estar associada à própria etiologia da depressão.

Na gama dos fatores associados ao surgimento da depressão estão os estilos atribucionais, os quais derivam do Modelo Reformulado de Desamparo Aprendido (Abramson, Seligman, \& Teasdale, 1978). A partir dessa perspectiva, eventos negativos e positivos são explicados através de estilos negativos ou positivos. Os estilos atribucionais negativos tendem a atribuir eventos negativos a causas internas (que dependem do sujeito), estáveis (pouco passiveis de mudanças) e globais (causa inespecifica). Por sua vez, os estilos atribucionais positivos tendem a, quando diante de eventos negativos, atribuir sua causalidade a fatores externos (causas que não dependem de si), temporárias (passiveis de modificação) e específicas (causa pontual) (Abramson et al., 1978). Assim, os estilos atribucionais negativos 
se associam ao desenvolvimento de sintomas depressivos, habilidades de enfrentamento menos favoráveis e piores condições de saúde física (Sanjuán \& Magallares, 2007).

Nesta pesquisa o desamparo foi outra variável de importante impacto sobre a pontuação intermediária e elevada na escala de depressão da BAID-IJ. Os adolescentes que pontuaram acima da mediana de desamparo exibiram pouco mais de duas vezes as chances de pertencer à faixa intermediária e aproximadamente 12 vezes mais chances de estar dentre aqueles com elevada pontuação na escala de depressão da BAID-IJ. Quanto maior o nível de desamparo do adolescente, mais propenso ele estava para apresentar niveis mais elevados de sintomas depressivos. Tais achados se assemelham aos encontrados em outros estudos realizados com adolescentes, que também observaram que o desamparo se associou a maiores niveis de depressão (Verma \& Gera, 2014). Um possivel caminho para a compreensão da relação encontrada entre o desamparo e a depressão em adolescentes pode estar associado ao próprio Modelo de Desamparo Aprendido (Abramson et al., 1978). Com isso, o individuo que apresenta niveis mais elevados de desamparo, consequentemente, tende a acreditar que pouco pode fazer para mudar o ambiente e as condições aversivas, provavelmente reduzindo a autoconfiança e a motivação para comportamentos mais adaptativos e ativos para saúde mental.

A respeito dos resultados referentes à solidão, os adolescentes que pontuaram acima da mediana exibiram aproximadamente três vezes mais chances de pertencer ao estrato intermediário e sete vezes ao de elevada pontuação. Em outros termos, quanto mais se vivenciou a solidão, maior a vulnerabilidade do adolescente à depressão, o que reforça que a solidão atua como significativo preditor para afetos e condições negativas em longo prazo (Jaremka et al., 2014). A solidão se relaciona à qualidade e à quantidade dos relacionamentos interpessoais e não necessariamente ao fato de a pessoa não ter pessoas por perto (Spithoven et al., 2016). Estudos mostram que a solidão atua como fator importante associado à depressão, mesmo após o controle dos sintomas depressivos (van Winkel et al., 2017), potencializa os riscos de desenvolver sintomas depressivos, transtornos de ansiedade e ideação suicida (Beutel et al., 2017), e se associou positiva e significativamente a sintomas de ansiedade e à depressão, o que significa dizer que quanto maiores os niveis de solidão, mais sintomas ansiosos e depressivos (Muyan, Chang, Jilani, Yu, Lin, \& Hirsch, 2016).

Em estudo brasileiro realizado com estudantes universitários, constatou-se que além de diferentes variáveis (sociodemográficas, outras psicopatologias e neuroticismo) terem contribuido para explicar o surgimento de sintomas depressivos, a solidão demonstrou ser um importante preditor da depressão, ainda que não tenha acrescentado grande variância explicativa, apenas $1 \%$ no modelo final (Barroso, Baptista, \& Zanon, 2018). Entre os adolescentes se observa correlação positiva com sintomas depressivos, bem como com problemas na saúde em geral e comportamentos de risco (uso de álcool e outras drogas). Uma possivel explicação para isso pode estar associada à forma como vivenciar a solidão interfere na capacidade de adaptação de adolescentes, influenciando, por exemplo, a prática de comportamentos mais desfavoráveis em saúde, que consequentemente poderiam interligar a solidão e piores niveis de saúde (Stickley et al., 2016).

Por fim, o último construto significativo foi a desesperança. Aqueles adolescentes que pontuaram acima da média em desesperança exibiram quase três vezes mais chances de pertencer ao estrato de elevada pontuação na depressão. Assim, quanto maior o nivel de desesperança, maior a chance de pontuar no nivel mais alto de sintomas depressivos. A provável justificativa para esses resultados parece estar associada à própria definição desse construto, que se relaciona às expectativas negativas quanto a não realização de resultados desejados e à expectativa de desamparo frente à ideia de irreversibilidade no que concerne a possibilidade de alteração de resultados aversivos (Abramson et al., 1978). Na literatura, níveis mais elevados de desesperança aparecem associados à depressão e ao suicídio, sendo que quanto mais insatisfeito com a vida, maiores as chances de apresentar 
niveis de moderado a grave de desesperança. Não apenas entre adolescentes, mas em outras fases do desenvolvimento, seja em amostras clínicas ou não clínicas, a desesperança mantém relação positiva com maior adoecimento mental (Waszczuk et al., 2016).

Em suma, observou-se que os fatores de risco (desamparo, desesperança e solidão) exibiram maior impacto preditivo em relação à elevada pontuação na escala de depressão da BAID-IJ, isso em comparação ao fator de proteção (autoconceito, $O R=5,7$ ), assim como às variáveis sociodemográficas (sexo e idade, respectivamente $O R$ = 6,1 e $O R=3,3$ ). Assim, constatou-se que os fatores de risco tiveram impacto superior à exposição a outros fatores, tais como o autoconceito e variáveis sociodemográficas. Além disso, constatou-se que, dentre os fatores protetivos, apenas o autoconceito se manteve no modelo final. De modo geral, acredita-se que esses achados podem auxiliar profissionais no rastreio de variáveis mais indicativas da vulnerabilidade ao desenvolvimento de sintomas depressivos significativos.

Como limitações, pontua-se, inicialmente, o modelo testado quanto à predição de sintomas depressivos entre adolescentes. Embora tenha apresentado capacidade preditiva de 70\% nesta amostra, acredita-se que futuramente possam ser analisadas outras variáveis importantes, como por exemplo, o otimismo disposicional e a esperança, as quais tendem a exibir efeito significativo frente à capacidade de adaptação às adversidades (ver Carver \& Scheier, 2014).

Um ponto a ser levado em consideração neste estudo, é o fato de que o modelo testado se baseia teoricamente no modelo de vulnerabilidade associado à depressão. Com isso, variáveis como a autoestima, quando em baixos niveis podem favorecer a maior predisposição a niveis mais elevados de sintomas depressivos. Por outro lado, a partir de um segundo modelo teórico chamado scar (cicatriz), entende-se que, por exemplo, niveis mais baixos de autoestima seriam uma consequência da depressão. Então, provavelmente as distorções cognitivas tão presentes nos transtornos depressivos, influenciariam negativamente niveis de variáveis positivas como a autoestima e o autoconceito, de modo a produzir verdadeiras "cicatrizes" e consequências a médio e longo prazo. Todavia, embora não testado nesse estudo, ambos os modelos teóricos não são excludentes, de modo que tanto baixos níveis de características como autoestima, podem atuar como fatores de risco ou proteção, quanto a depressão pode afetar a diminuição dos niveis dessas variáveis, o que corresponde a um modelo de relação reciproca (Orth \& Robins, 2013).

Outra limitação corresponde à amostra de conveniência e não probabilistica, uma vez que não permite a generalização dos achados para a população adolescente de modo mais amplo. Recomenda-se que futuros estudos utilizem critérios de randomização (por exemplo, de participantes, salas de aula ou instituições), visando a representatividade em um determinado contexto, seja ele um conjunto de escolas ou mesmo na comunidade. Salienta-se, também, que as diferenças observadas, principalmente quanto ao sexo feminino, e a maior predisposição aos sintomas depressivos mais elevados, devem ser analisadas com parcimônia, em especial, frente às questões amostrais discutidas anteriormente. Ademais, hipóteses e explicações para a prevalência do sexo feminino no que tange os níveis de depressão igualmente é um ponto de debate entre pesquisadores brasileiros, ao passo que ressalta a importância de fatores genéticos e biopsicossociais uma vez que a depressão é causada por aspectos multifatoriais (Baptista, Baptista, \& Oliveira, 1999)

Finalmente, espera-se que estes resultados gerem implicações para a forma como profissionais da psicologia da saúde, escolar ou clínica venham a atuar frente à depressão na adolescência, uma vez que ao identificar fatores geradores de maior risco, algumas ações podem ser direcionadas. Por exemplo, no âmbito escolar, poderiam ser realizadas ações interventivas (em grupo ou individuais) baseadas nos fatores de maior impacto explicativo de sintomas depressivos na adolescência, ou seja, procurar desenvolver estratégias mais adaptativas para lidar com a sensação de desamparo, a desesperança e a solidão, quando do enfrentamento de adversidades excepcionais ou cotidianas. É importante considerar a necessi- 
dade de trabalhar com estratégias de intervenção pautadas em evidências, cujo intuito principal seja o de apontar variáveis que influenciam significativamente a capacidade de adaptação dos adolescentes frente às adversidades.

\section{Referências}

Abramson, L. Y., Seligman, M. E. P., \& Teasdale, D. C. (1978). Learned helplessness in humans: Critique and reformulation. Journal of Abnormal Psychology, 87, 49-74.

Barroso, S. M., Baptista, M. N., \& Zanon, C. (2018). Solidão como variável preditora na depressão em adultos. Estudos Interdisciplinares em Psicologia, 9(3 supl.), 26-37. https://doi.org/10.5433/2236-6407.2018vgn3suplp26

Baptista, M. N., Baptista, A, S. D., \& Oliveira, M. G. (1999). Depressão e gênero: Por que as mulheres deprimem mais que os homens? Temas em psicologia, 7(2), 143-156.

Beck, A. T. (2008). The evolution of the Cognitive Model of Depression and its neurobiological correlates. American Journal of Psychiatry, 165(8), 969-977.

Beutel, M. E., Klein, E. M., Brähler, E., Reiner, I., Jünger, C., Michal, M., ... Tibubos, A. N. (2017). Loneliness in the general population: Prevalence, determinants and relations to mental health. BMC Psychiatry, 17(97). https:// doi.org/10.1186/s12888-017-1262-X

Borges, L. S., Baptista, M. N., \& Serpa, A. L. O. (2015). Bateria de Avaliação de Indicadores de Depressão Infantojuvenil (BAID-IJ). (Relatório técnico não publicado, Universidade São Francisco, Itatiba, SP, Brasil).

Cardoso, C. (2018). Propriedades psicométricas da Bateria de Avaliação de Indicadores da Depressão Infantojuvenil (BAID-IJ). (Tese de doutorado, Universidade São Francisco, Campinas). Recuperado de https://Www.usf. edu.br/galeria/getlmage/427/14117630280977079.pdf

Carver, C. \& Scheier, M. F. (2014). Dispositional optimism. Trends in Cognitive Sciences, 18(6), 293-299. https://doi. org/10.1016/j.tics.2014.02.003.

Cho, S. M. \& Shin, Y. M. (2013). The promotion of mental health and the prevention of mental health problems in child and adolescent. Korean Journal of Pediatrics, 56(11), 459-464. https://doi.org/10.3345/kjp.2013.56.11.459

Das, J. K., Salam, R. A., Lassi, Z. S., Khan, M. N., Mahmood, W., Patel, V., \& Bhutta, Z. A. (2016). Interventions for adolescent mental health: An overview of systematic reviews. Journal of Adolescent Health, 59(4), S49-S60. https://doi.org/10.1016/j.jadohealth.2016.06.020

Essau, C. A., Lewinsohn, P. M., Seeley, J. R., \& Sasagawa, S. (2010). Gender differences in the developmental course of depression. Journal of Affective Disorders, 127, 185-190. https://doi.org/10.1016/j.jad.2010.05.016

Freitas, C. P. P. (2016). Relação das caracteristicas pessoais positivas com o bem-estar. (Tese de doutorado, Instituto de Psicologia, Universidade Federal do Rio Grande do Sul, Porto Alegre). Recuperado de http:// www.lume.ufrgs.br/handle/10183/147072
Germain, F. \& Marcotte, D. (2016). Sintomas de depressão e ansiedade na transição do ensino secundário ao ensino médio: Evolução e fatores influentes. Adolescência e Saúde, 13(1), 19-28.

Grolli, V., Wagner, M. F., \& Dalbosco, S. N. P. (2017). Sintomas depressivos e de ansiedade em adolescentes do Ensino Médio. Revista de Psicologia da IMED, 9(1), 87103. https://doi.org/10.18256/2175-5027.2017.vgi1.2123

Hodes, G. E., Walker, D. M., Labonté, D., Nestler, E. J., \& Russo, S. J. (2016). Understanding the epigenetic basis of sex differences in depression. Journal of Neuroscience Research, 95, 692-702. https://doi.org/10.1002/jnr.23876

Jaremka, L. M., Andridge, R. R., Fagundes, C. P., Alfano, C. M., Povoski, S. P., Lipari, A. M., ... Kiecolt-Glaser, J. K. (2014). Pain, depression, and fatigue: Loneliness as a longitudinal risk factor. Health Psychology, 33(9), 948957. https://doi.org/10.1037/a0034012

Kaushik, A., Kostaji, E., \& Kyriakopoulos, M. (2016). The stigma of mental illness in children and adolescents: A systematic review. Psychiatry Research, 243, 469-494. https://doi.org/10.1016/j.psychres.2016.04.042

Luz, R. T., Coelho, E. A. C., Teixeira, M. A., Barros, A. R., Carvalho, M. F. A. A., Almeida, M. S. (2018). Saúde mental como dimensão para o cuidado de adolescentes. Revista Brasileira de Enfermagem, 71(supl. 5), 2087-2093. https://doi.org/10.1590/0034-7167-2016-0192

Luz, J. M. O., Murta, S. G., \& Aquino, T. A. A. (2015). Programas de promoção de saúde mental em grupo para adolescentes. In C. B. Neufeld (Org.), Terapia Cognitivo-Comportamental em grupo para crianças e adolescentes (pp. 52-72). Porto Alegre: Artmed.

Matthews, T., Danese, A., Wertz, J., Odgers, C. L., Ambler, A., Moffitt, T. E., \& Arseneault, L. (2016). Social isolation, loneliness and depression in young adulthood: A behavioural genetic analysis. Social Psychiatry and Psychiatric Epidemiology, 51(3), 339-348. https://doi.org/10.1007/ s00127-016-1178-7

Maughan, B., Collishaw, S., \& Stringaris, A. (2013). Depression in Childhood and Adolescence. Journal of the Canadian Academy of Child and Adolescent Psychiatry, 22(1), 35-40.

McGuire, T. C., McCormick, K. C., Koch, M. K., \& Mendle, J. (2019). Pubertal maturation and trajectories of depression during early adolescence. Frontiers in Psychology, 10. 1-7. https://doi.org/10.3389/fpsyg.2019.01362

Muyan, M., Chang, E. C., Jilani, Z., Yu, T., Lin, J., \& Hirsch, J. K. (2016). Loneliness and negative affective conditions in adults: Is there any room for hope in predicting anxiety and depressive symptoms? The Journal of Psychology, 150(3), 333-41. https://doi.org/10.1080/00223980.2015.1039474

Orth, U., \& Robins, R. W. (2013). Understanding the link between low self-esteem and depression. Current Directions in Psychological Science, 22(6), 455-460. https:// doi.org/10.1177/0963721413492763.

Paixão, R. F., Patias, N. D., \& Dell'Aglio, D. D. (2018). Self-esteem and symptoms of mental disorder in the adolescence: Associated variables. Psicologia: Teoria e Pesquisa, 34, e34436, 1-8. https://doi.or$\mathrm{g} / 10.1590 / 0102.3772 \mathrm{e} 34436$ 
Reardon, T., Harvey, K., Baranowska, M., O'Brien, D., Smith, L., \& Creswell, C. (2017). What do parents perceive are the barriers and facilitators to accessing psychological treatment for mental health problems in children and adolescents? A systematic review of qualitative and quantitative studies. European Child \& Adolescent Psychiatry, 26, 623-647. https://doi.org/10.1007/s00787-016-0930-6

Robles-Piña, R. A. (2011). Depression and self-concept: Personality traits or coping styles in reaction to school retention of hispanic adolescentes. Depression Research and Treatment, 2011, 1-8. https://doi. org/10.1155/2011/151469

Sagar, R. \& Krishnan, V. (2017). Preventive strategies in child and adolescent psychiatry. Indian Journal of Social Psychiatry, 33, 118-122. https://doi.org/10.4103/ijsp.ijsp_43_17

Sanjuán, P., \& Magallares, A. (2007). Estilos explicativos y estrategias de afrontamiento. Clínica y Salud, 18(1), 83-98.

Salk, R. H., Hyde, J. S., \& Abramson, L. Y. (2017). Gender differences in depression in representative national samples: Meta-analyses of diagnoses and symptoms. Psychological Bulletin, 143(8), 783-822. https://doi. org/10.1037/buloo00102

Salk, R. H., Petersen, J. L., Abramson, L. Y., \& Hyde, J. S. (2016). The contemporary face of gender differences and similarities in depression throughout adolescence: Development and chronicity. Journal of Affective Disorders, 205, 28-35. https://doi.org/10.1016/j.jad.2016.03.071

Shavelson, R. J., \& Bolus, R. (1982). Self-Concept: The interplay of theory and methods. Journal of Educational Psychology, 74(1), 3-17.

Spithoven, A. W. M., Lodder, G. M. A., Goossens, L., Bijttebier, P., Bastin, M., Verhagen, M., \& Scholte, R. H. J. (2016). Adolescents' loneliness and depression associated with friendship experiences and well-being: A person-centered approach. Journal Youth Adolescence, 46, 429-441 https://doi.org/10.1007/s10964-016-0478-2

Stickley, A., Koyanagi, A., Koposov, R., Blatný, M., Hrdlička, M., Schwab-Stone, M., \& Ruchkin, V. (2016). Loneliness and its association with psychological and somatic health problems among Czech, Russian and U.S. adolescents. BMC Psychiatry, 16(128), 1-11. https:// doi.org/10.1186/s12888-016-0829-2

van Winkel, M., Wichers, M., Collip, D., Jacobs, N., Derom, C., Thiery, E., ... Peeters, F. (2017). Unraveiling the role of loneliness in depression: The relationship between daily life experience and behavior. Psychiatry, 80(2), 104-117. https://doi.org/10.1080/00332747.2016.1256143

Verma, S. \& Gera, M. (2014). Learned helplessness in adolescents. International Journal of Science and Research (IJSR), 3(7), 930-932.

Waszczuk, M. A., Coulson, A. E., Gregory, A. M., \& Eley, T. C. (2016). A longitudinal twin and sibling study of the hopelessness theory of depression in adolescence and young adulthood. Psychological Medicine, 46, 1935-1949. https://doi.org/10.1017/S0033291716000489

World Health Organization (WHO). (2012). Adolescent mental health: Mapping actions of nongovernmental organizations and other international development organizations. Geneva, Suiça.
World Health Organization (WHO). (2017). Depression and Other Common Mental Disorders: Global Health Estimates. Geneva, Suiça.

World Health Organization (WHO). (2014). Health for the World's Adolescents A second chance in the second decade. Geneva, Suiça.

Yang, X., Lau, J. T. F., \& Lau, M. C. M. (2018). Predictors of remission from probable depression among Hong Kong adolescents - A large-scale longitudinal study. Journal of Affective Disorders, 229, 491-497. https://doi. org/10.1016/j.jad.2017.12.080

\section{Laís Santos-Vitti}

Mestre em Psicologia Social pela Universidade Federal de Sergipe (UFS), em São Cristóvão, SE, Brasil; doutoranda em Psicologia pelo Programa de Pós-Graduação em Psicologia Stricto Sensu da Pontifícia Universidade Católica de Campinas (PUC-Camp), Campinas, SP, Brasil.

\section{André Faro}

Doutor em Psicologia pela Universidade Federal da Bahia (UFBA), em Salvador, BA, Brasil; professor do Programa de Pós-Graduação Stricto Sensu em Psicologia da Universidade Federal de Sergipe (UFS), São Cristóvão, SE, Brasil.

\section{Makilim Nunes Baptista}

Doutor em Psiquiatria e Psicologia Médica pela Universidade Federal de São Paulo (UNIFESP), em São Paulo, SP, Brasil; professor do Programa de Pós-Graduação Stricto Sensu em Psicologia da Universidade São Francisco (USF), em Campinas, SP, Brasil.

\section{Endereço para correspondência}

Lais Santos

Pontifícia Universidade Católica de Campinas

Programa de Pós-Graduação em Psicologia

Av. John Boyd Dunlop, s/n., Prédio Administrativo Centro de Ciências da Vida

Jd. Ipaussurama, 13060-904

Campinas, SP, Brasil 